\title{
Influence of material properties on extraordinary optical transmission through hole arrays
}

\author{
Sergio G. Rodrigo, ${ }^{1}$ F. J. García-Vidal, ${ }^{2}$ and L. Martín-Moreno ${ }^{1}$ \\ ${ }^{1}$ Departamento de Física de la Materia Condensada, Facultad de Ciencias-ICMA, Universidad de Zaragoza-CSIC, \\ E-50009 Zaragoza, Spain \\ ${ }^{2}$ Departamento de Física Teórica de la Materia Condensada, Universidad Autónoma de Madrid, Madrid 28049, Spain
}

(Received 5 November 2007; published 4 February 2008)

\begin{abstract}
We present a theoretical study, based on the finite difference time domain method, of the optical response of circular hole arrays drilled in several metal films ( $\mathrm{Ag}, \mathrm{Au}, \mathrm{Cu}, \mathrm{Al}, \mathrm{Ni}, \mathrm{Cr}$, and $\mathrm{W})$. Two series of structures are studied. In the first one, transmittance peaks are analyzed as all geometrical parameters defining the system are scaled, except for the metal thickness which is kept constant, showing good agreement with existing experimental data. In the second series, the metal thickness is also scaled. This allows a clear distinction in the behavior of different metals: $\mathrm{Ag}, \mathrm{Au}$, and $\mathrm{Cu}$ show even larger transmittance peaks than hole arrays in a perfect conductor with the same nominal parameters. This is due to both a larger effective hole area and smaller absorption. In the case of $\mathrm{Ni}$ and $\mathrm{Cr}$, the transmittance is much smaller due to absorption. Band structure calculations confirm that surface electromagnetic modes sustained by the perforated metal film are responsible for the extraordinary optical transmission phenomenon.
\end{abstract}

DOI: 10.1103/PhysRevB.77.075401 PACS number(s): 42.25.Bs, 73.20.Mf, 42.79.Dj, 78.66.Bz

\section{INTRODUCTION}

Extraordinary optical transmission (EOT), ${ }^{1}$ i.e., resonant peaks in the transmission of light through subwavelength holes drilled in a metal film, has attracted a great deal of attention from the scientific community in the past years due to both its fundamental interest and its potential for applications. $^{2}$

Pioneering attempts to understand EOT pointed out surface plasmon polariton (SPP) modes ${ }^{1,3-5}$ as responsible for the phenomenon. More generally, it has been shown that EOT-like behavior occurs whenever two surface modes are coupled between themselves and weakly coupled to a continuum, ${ }^{3}$ allowing a Fano-like description of the process. ${ }^{6}$ Examples of this general mechanism are the cases of wave transmission aided by Brewster-Zenneck waves in hole arrays drilled in tungsten, ${ }^{7}$ guide cavity modes on slit arrays covered by a dielectric layer, ${ }^{8}$ surface electromagnetic waves in photonic crystals, ${ }^{9-11}$ surface sonic waves, ${ }^{12}$ and even the transmission of cold atoms through optically induced potential barriers. ${ }^{13}$

Going back to the case of two-dimensional hole arrays (2DHAs), many studies have been devoted to study the dependence of EOT on the different parameters defining the system. As regards geometrical parameters, it has been found that the hole shape can strongly influence both the polarization properties and the intensity of the transmission. This has been related to the presence of single-hole transmission resonances that couple to the SPPs. ${ }^{14-19}$

With respect to the material properties, it is known that some metals (notably $\mathrm{Au}$ and $\mathrm{Ag}$ ) are best suited for EOT than others $\left(\mathrm{Ni}, \mathrm{Co}\right.$, etc.). ${ }^{1,20}$ However, a systematic comparative between different metals was lacking until recent experiments performed by Przybilla et al. were reported. ${ }^{21}$

These experiments analyzed EOT through 2DHA made of circular holes drilled in optically thick metal films, deposited on a glass substrate. The study considered different metals and analyzed the peak transmittance as a function of lattice parameter which, in turn, controlled the resonant wavelength. If the system were a perfect electric conductor (PEC), the transmission would not depend on the lattice parameter, provided all length scales in the system are scaled in the same way. Therefore, deviations from this behavior reflects the effect of material properties. In the experiments, ${ }^{21}$ the hole radii were scaled with the lattice parameter but, due to practical limitations, the metal thickness was kept constant, which makes the analysis even more complex. Another possible complication is that finite size effects, surface quality, and imperfections in hole shape in a real system could depend on the metal considered.

In this work, we present a theoretical study of EOT in periodic hole arrays drilled in different metals. First, we compare with the experimental results presented in Ref. 21. The excellent agreement found with the experimental data validates our approach and, at the same time, shows that finite size effects and disorder have minimal relevance in the performed experiments. Additionally, we present a study in which all lengths are scaled with the lattice parameter. In both cases, comparison with the PEC case helps as understand the effect of material properties in the light transmission through 2DHA.

\section{THEORETICAL APPROACH}

Our calculations are performed with the finite difference time domain (FDTD) method. ${ }^{22,23}$ Infinite periodic 2DHAs are simulated by applying Bloch conditions at the boundaries of the unit cell and imposing "uniaxial perfect matched layers" 22 at surfaces parallel to the metal film. In the FDTD method, Maxwell's equations are discretized in both space and time. Therefore, convergence depends on both mesh size and temporal step. In order to properly calculate the influence of material properties, the rapidly decaying fields inside the metal should be accurately computed. This, together with the proper representation of circular holes in Cartesian coordinates (which are the natural choice for square arrays in a 
TABLE I. Dielectric constant fitted parameters of $\mathrm{Ag}, \mathrm{Au}, \mathrm{Cu}, \mathrm{Al}, \mathrm{Ni}, \mathrm{Cr}$, and $\mathrm{W} . \omega_{P}, \gamma, \Gamma$, and $\Omega$ are in $\mathrm{eV}$.

\begin{tabular}{|c|c|c|c|c|c|c|c|}
\hline & $\mathrm{Ag}$ & $\mathrm{Au}$ & $\mathrm{Cu}$ & $\mathrm{Al}$ & $\mathrm{Ni}$ & $\mathrm{Cr}$ & W \\
\hline$\epsilon_{r}$ & 4.6 & 5.967 & 1.0 & 1.0 & 1.0 & 1.0 & 1.0 \\
\hline$\omega_{P 0}$ & 9.0 & 8.729 & 8.212 & 10.83 & 4.621 & 4.406 & 5.955 \\
\hline$\gamma_{0}$ & 0.07 & 0.065 & 0.03 & 0.047 & 0.021 & 0.047 & 0.027 \\
\hline$\omega_{P 1}$ & & & & & 6.929 & & 2.286 \\
\hline$\gamma_{1}$ & & & & & 1.771 & & 0.335 \\
\hline$\omega_{P 2}$ & & & & & 7.062 & & \\
\hline$\gamma_{2}$ & & & & & 3.443 & & \\
\hline$\Gamma_{0}$ & 1.2 & 0.433 & 0.378 & 0.333 & 1.021 & 3.175 & 0.590 \\
\hline$\Omega_{0}$ & 4.9 & 2.684 & 0.291 & 0.162 & 1.458 & 0.121 & 0.984 \\
\hline$\Delta \epsilon_{0}$ & 1.10 & 1.09 & 84.49 & 1940.97 & 2.1 & 1191.85 & 12.0 \\
\hline$\Gamma_{1}$ & & & 1.056 & 0.312 & 2.410 & 1.305 & 1.653 \\
\hline$\Omega_{1}$ & & & 2.957 & 1.544 & 3.443 & 0.543 & 2.066 \\
\hline$\Delta \epsilon_{1}$ & & & 1.395 & 4.706 & 1.2 & 58.79 & 14.4 \\
\hline$\Gamma_{2}$ & & & 3.213 & 1.351 & & 2.676 & 2.479 \\
\hline$\Omega_{2}$ & & & 5.3 & 1.808 & & 1.970 & 4.132 \\
\hline$\Delta \epsilon_{2}$ & & & 3.018 & 11.39 & & 34.21 & 12.9 \\
\hline$\Gamma_{3}$ & & & 4.305 & 3.382 & & 1.335 & \\
\hline$\Omega_{3}$ & & & 11.18 & 3.473 & & 8.775 & \\
\hline$\Delta \epsilon_{3}$ & & & 0.598 & 0.558 & & 1.238 & \\
\hline
\end{tabular}

film), impose very small mesh sizes. In our calculation, we use mesh sizes ranging from 2 to $5 \mathrm{~nm}$. The dielectric constant in cells at the metal-dielectric interface is taken as that of the medium with the largest volume inside that particular cell. We have checked that transmittance spectra obtained in this manner virtually coincide with those obtained with a modal expansion, ${ }^{24}$ in the case of 2DHA in PEC for which the latter method is exact.

For the calculation of the transmittance, the structures were excited by a Gaussian wave packet composed of normally incident plane waves (with the electric field pointing along one of the axes of the square array) and all frequencies of interest. Spectra were calculated after projection onto diffracted modes. In the comparison with experimental data, only the zero order mode was considered in the postprocessing, as experimental intensities were collected in a small solid angle centered around the normal direction. Additionally, in our study we will correlate transmittance features to the dispersion relation of modes in the corrugated slab. For this, the band structure is calculated by exciting the system with a superposition of Bloch waves with a well defined crystal momentum and imposing Bloch's theorem at the boundaries, as done in Ref. 25.

Metals require a special treatment in FDTD method, as the dielectric constant is local in frequency domain but nonlocal in the time domain. We have used the "piece linear recursive convolution method," 22,23 which can efficiently treat dispersive media, provided their dielectric constant $\epsilon(\omega)$ can be expressed as a sum of Drude and Lorentz terms:

$$
\epsilon(\omega)=\epsilon_{r}-\sum_{j} \frac{\omega_{P j}^{2}}{\omega\left(\omega+\iota \gamma_{j}\right)}-\sum_{j} \frac{\Delta \epsilon_{j} \Omega_{j}^{2}}{\omega^{2}-\Omega_{j}^{2}+\iota \omega \Gamma_{j}} .
$$

We have considered the following materials: $\mathrm{Ag}, \mathrm{Au}, \mathrm{Cu}$, $\mathrm{Al}, \mathrm{Ni}, \mathrm{Cr}$, and $\mathrm{W}$, taking the values for the parameters in the Drude-Lorentz form either from the literature (when available) $)^{26,27}$ or from fits to data in Palik's handbook. ${ }^{28,29}$ The fitting parameters used are given in Table I, and the wavelength dependence of the dielectric constant obtained from them is depicted in Fig. 1 for reference. Also, for future reference, Fig. 2 renders both the skin depth, $\delta=\left[\operatorname{Im}\left(k_{m}\right)\right]^{-1}$ (with $k_{m}^{2}=\epsilon_{m} \omega^{2} / c^{2}$ ) and the SPP absorption length, $L_{S P P}$ $=\left[2 \operatorname{Im}\left(k_{S P P}\right)\right]^{-1}$, with $k_{S P P}=(\omega / c)\left(\epsilon_{S} \epsilon_{m} /\left(\epsilon_{S}+\epsilon_{m}\right)\right)^{1 / 2}$ the SPP longitudinal wave vector. Here, $\epsilon_{m}$ and $\epsilon_{S}$ are the metaldielectric constant and the substrate dielectric constant, respectively. We stress here that the dielectric constants used in this study are taken directly from experimental data on bulk. No attempt has been made to improve the comparison between computed and experimental transmission spectra by incorporating additional fitting parameters.

\section{EXTRAORDINARY OPTICAL TRANSMISSION PEAK RELATED TO THE METAL-SUBSTRATE SURFACE PLASMON}

\section{A. Peak position}

Figure 3 renders the FDTD results for the transmittance spectra of an array (lattice parameter $P=400 \mathrm{~nm}$ ) of circular holes with diameter $d=P / 1.75=229 \mathrm{~nm}$ in different metal films. In all cases, the metal thickness is $w=250 \mathrm{~nm}$, and the films are deposited on a substrate with dielectric constant $\epsilon_{S}=2.25$. The figure clearly shows the difference in transmittance spectra between different metals. We also include the calculation for $\mathrm{W}$ which, in the considered frequency range, 

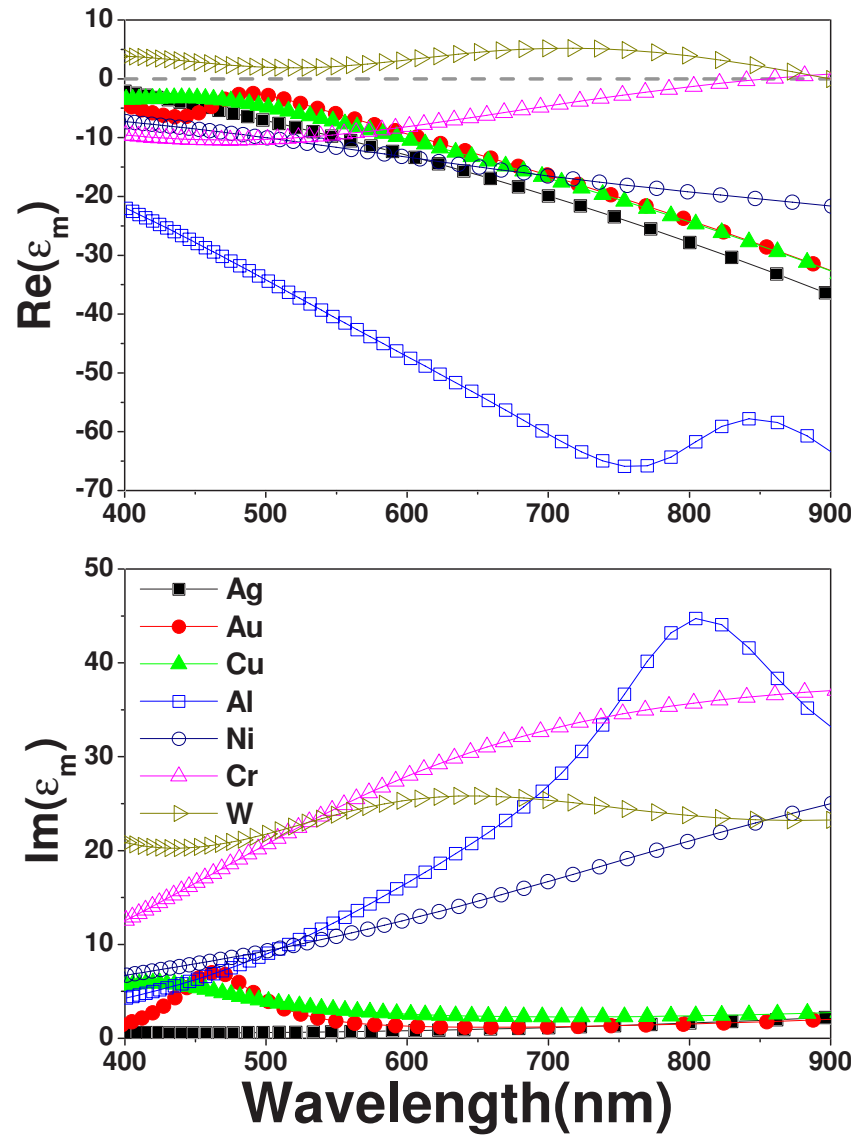

FIG. 1. (Color online) Dielectric constant for $\mathrm{Ag}, \mathrm{Au}, \mathrm{Cu}, \mathrm{Al}$, $\mathrm{Ni}, \mathrm{Cr}$, and $\mathrm{W}$ as a function of the wavelength (Table I).

is a dielectric, i.e., $\operatorname{Re}\left(\epsilon_{\mathrm{W}}\right)>0$. Notice that the heights of EOT peaks in $\mathrm{W}$ are even smaller than those of the "bad" metals $(\mathrm{Ni}$ and $\mathrm{Cr}$ ). Notice also that $\mathrm{W}$ has a transmission maximum very close to the Rayleigh condition, $\lambda_{R}=\sqrt{\epsilon_{S}} P$, wavelength at which the $(1,0)$ diffraction order changes character from evanescent to radiative, while the corresponding one in the metallic case is redshifted (by as much as $\approx 125 \mathrm{~nm}$ in the case of the "good" metals in the optical regime: $\mathrm{Ag}, \mathrm{Au}$, and $\mathrm{Cu}$ ).

In the rest of this paper and following Ref. 21, EOT is characterized by the transmittance peak appearing close to $\lambda_{R}$. More precisely, in the metallic case, this peak is related to the spectral location of the SPP of the corrugated structure. ${ }^{3,21,30}$ Here, we will label this peak as $S_{1,0}$. Figure 4 shows the $S_{1,0}$ peak spectral position (defined as $\lambda_{1,0}$ ) as a function of the period for the parameters considered in Ref. $21(w=250 \mathrm{~nm} ; d=P / 1.75)$. In addition, Fig. 4 also renders the light line in the substrate (continuous line) and the results obtained by considering the metal as a PEC, i.e., a metal with $\epsilon=-\infty$, with the same nominal parameters (asterisk data points). Notice that a flat metal surface does not support surface electromagnetic (EM) modes, but a periodically corrugated one behaves as if it had a Drude-like dielectric response in which the effective plasma frequency depends only on the geometrical parameters. ${ }^{31-33}$ Thus, comparison with the PEC case allows as to discern geometrically induced effects from the ones due to material properties. In any case,
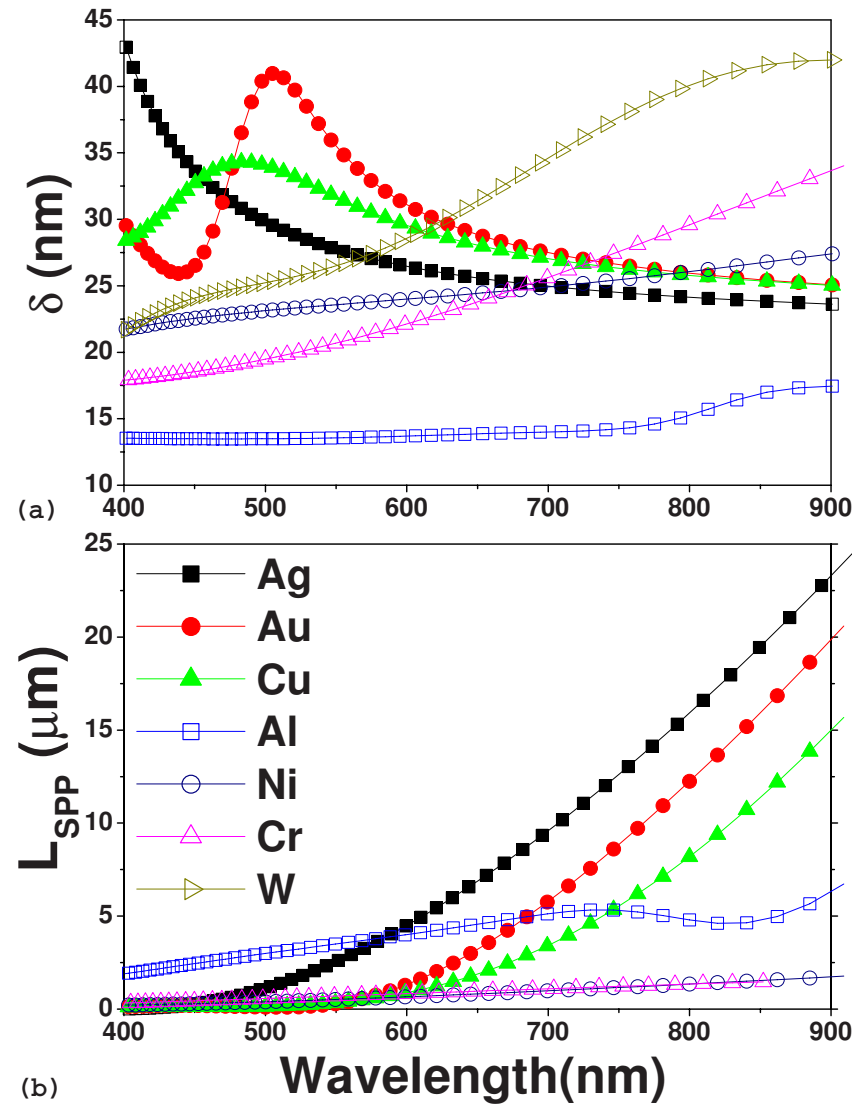

FIG. 2. (Color online) Spectral dependence for different metals of (a) skin depth for a plane wave impinging at normal incidence on the metal surface and (b) absorption length for SPP on the metaldielectric interface.

the agreement between these calculations and the experimental measurements (see Fig. 3 in Ref. 21) is remarkable. The small differences could be attributed to variations in the actual dielectric constant from the bulk value to finite size effects $^{34}$ and/or to irregularities on hole shape and size related to the small uncertainties from focused ion beam lithography technique (which has an accuracy of the order of $10 \mathrm{~nm}$ ). In our opinion, the agreement obtained validates FDTD calculations as a predictive tool in this kind of systems. These results also confirm that, in the experiments considered, the effect of possible inhomogeneities in hole shape and size was not relevant. Notice that the good agreement with the experimental data was obtained by using the bulk dielectric constant, despite the fact that the dielectric properties at the surface could have been modified by the processing related to drilling of the holes.

In order to obtain further insight on the origin of EOT phenomena, Fig. 5 renders the dependence with period of $\lambda_{1,0}$ for the metals $\mathrm{Ag}$ and $\mathrm{Al}$ and also for W. In this figure, we have also included the spectral location of the minimum which appears to be associated with the $S_{1,0}$ peak, slightly blue shifted from the maximum. Results are presented for the case of constant film thickness $w=250 \mathrm{~nm}$. In the same figure, we represent both $\lambda_{R}$ and the folded dispersion relation for the SPP of a flat metal-dielectric interface [given by the expression $\left.(\omega / c)\left(\epsilon_{S} \epsilon_{m} /\left(\epsilon_{S}+\epsilon_{m}\right)\right)^{1 / 2}=2 \pi / P\right]$. In the case of 

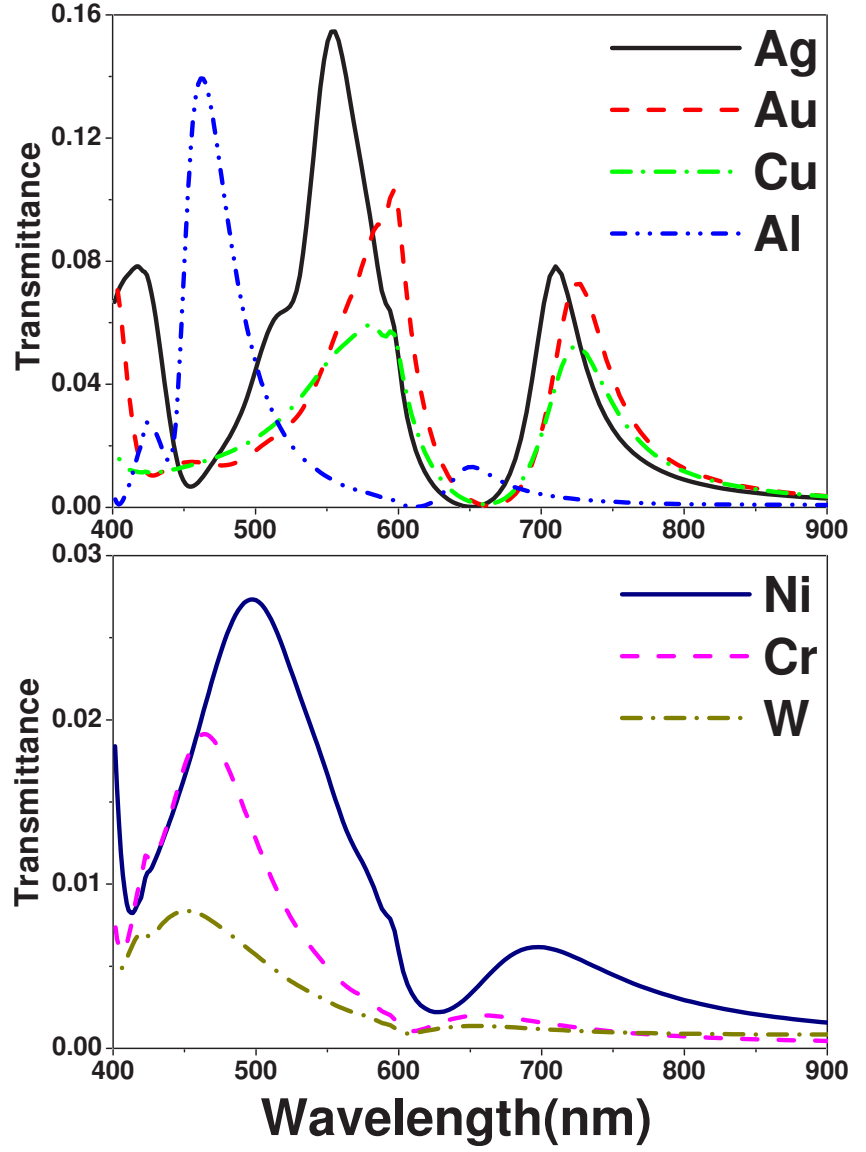

FIG. 3. (Color online) A representative case of computed transmission spectra for the different metals considered. The geometrical parameters defining the array are period $P=400 \mathrm{~nm}$, metal thickness $w=250 \mathrm{~nm}$, and hole diameter $d=P / 1.75$.

metals, the correspondence between transmittance minima and SPP of the flat surface (with no holes) is evident from the figure, with the maxima following the same trend at slightly longer wavelengths. In the case of the dielectric (W),

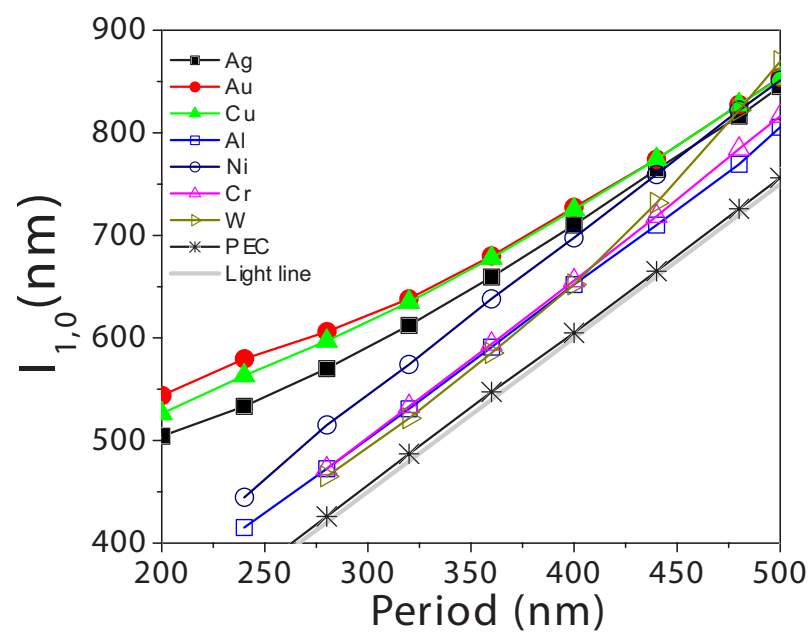

FIG. 4. (Color online) $(1,0)$ substrate peak position $\lambda_{1,0}$ as a function of the array period both for the metals investigated and for a perfect electrical conductor. Hole radius is scaled with the period as $d=P / 1.75$ but film thickness is kept constant at $w=250 \mathrm{~nm}$.
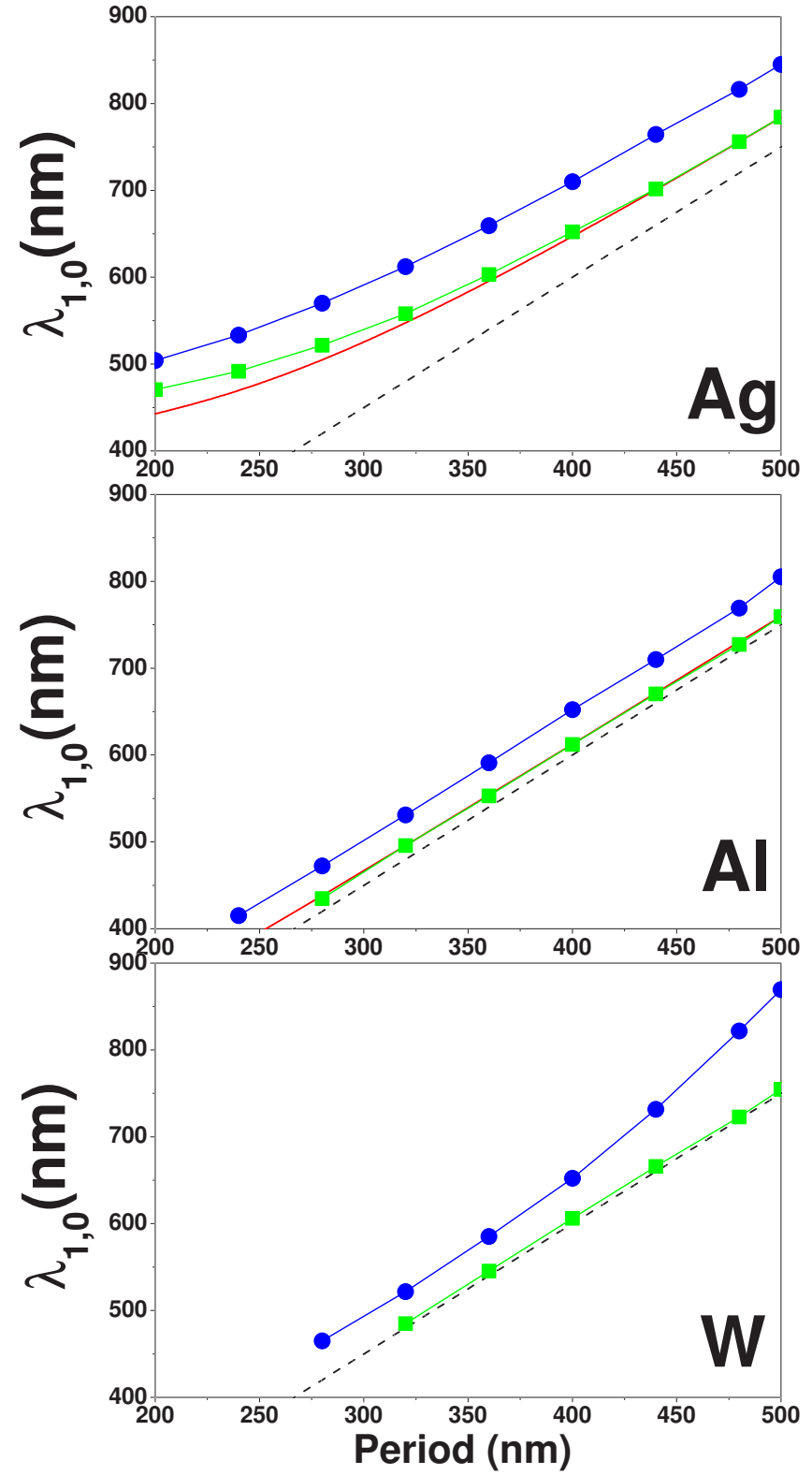

FIG. 5. (Color online) Dependence on period of signatures related to the $(1,0)$ substrate peak: maximum (circular symbols) and minimum (square symbols). Hole radius is scaled with the period as $d=P / 1.75$ but film thickness is kept constant at $w=250 \mathrm{~nm}$. The solid red line represents the SPP dispersion relation for a flat metaldielectric interface, while the dashed black line corresponds to the dielectric light line.

both maximum and minimum have much smaller amplitudes with respect to the transmission background than in the case of metals (see Fig. 3), with the very weak minimum appearing at $\lambda_{R}$.

Both maximum and minimum are related to the surface EM modes of the corrugated structure. More precisely, each surface EM mode has associated maximum and minimum transmissions, characterized by a resonant Fano-like function times a smooth function related to the coupling of the incident wave with the surface mode. At normal incidence, the incident wave can couple to SPPs in the periodically corru- 


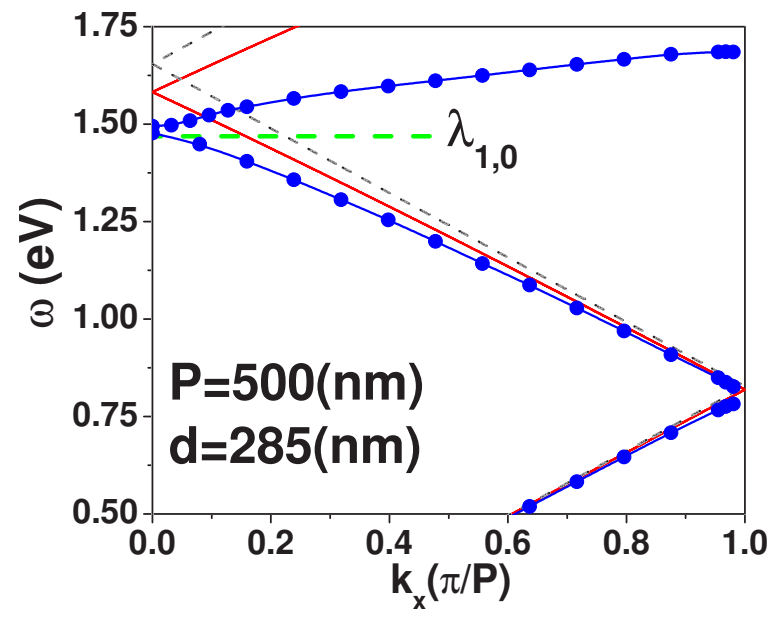

FIG. 6. (Color online) SPP dispersion relation in the $\Gamma-X$ direction of the first Brillouin zone for the dielectric-silver interface. Circular symbols (blue line) show the FDTD band structure for a 2DHA with period $P=500 \mathrm{~nm}$ and metal thickness $w=250 \mathrm{~nm}$. Flat surface SPP dispersion relation is depicted with a solid red line. Dashed black line represents the light line. The spectral position of the $(1,0)$ transmission maximum, $\lambda_{1,0}$, is depicted by a dashed horizontal green line.

gated structure which originate from plane waves differing from $k_{\|}=0$ by a reciprocal lattice vector. This is confirmed by Fig. 6, which renders the folded light line, the folded SPP dispersion relation of the flat metal-dielectric interface, and the FDTD calculation for the band structure of surface EM modes of the corrugated surface.

\section{B. Transmission intensity}

Up to here, we have concentrated on the dependence of the spectral position of transmission anomalies with the period of the array. The results for the peak intensities are presented in Fig. 7, which renders the maximum transmittance of the $S_{1,0}$ peak, $T_{1,0}$, as a function of its spectral position (i.e., for different periods) for several materials. In panel (a), all lengths defining the system are scaled with the period, except for the metal thickness, which is kept constant at $w$ $=250 \mathrm{~nm}$ (this is the case considered in Ref. 21). In panel (b), all lengths are scaled with the period. In this latter case, if the metal were a PEC, the peak transmittance would not depend on period (line with asterisks). A real metal presents two main differences with respect to a PEC, each of them having an opposite effect on the transmittance. On the one hand, a real metal absorbs energy, which reduces the transmittance. This is more apparent in resonant processes, which require the EM field to stay for a longer time at the surface. As this "resonance" time is inversely proportional to the peak width, absorption has a larger influence on the narrowest transmittance peaks. On the other hand, the EM field penetrates in a real metal, effectively increasing the hole area accessible to the field, and therefore increasing the transmittance. Several approximations can be envisaged in order to take into account the effective hole area. For instance, the hole can be considered as a finite portion of a waveguide.

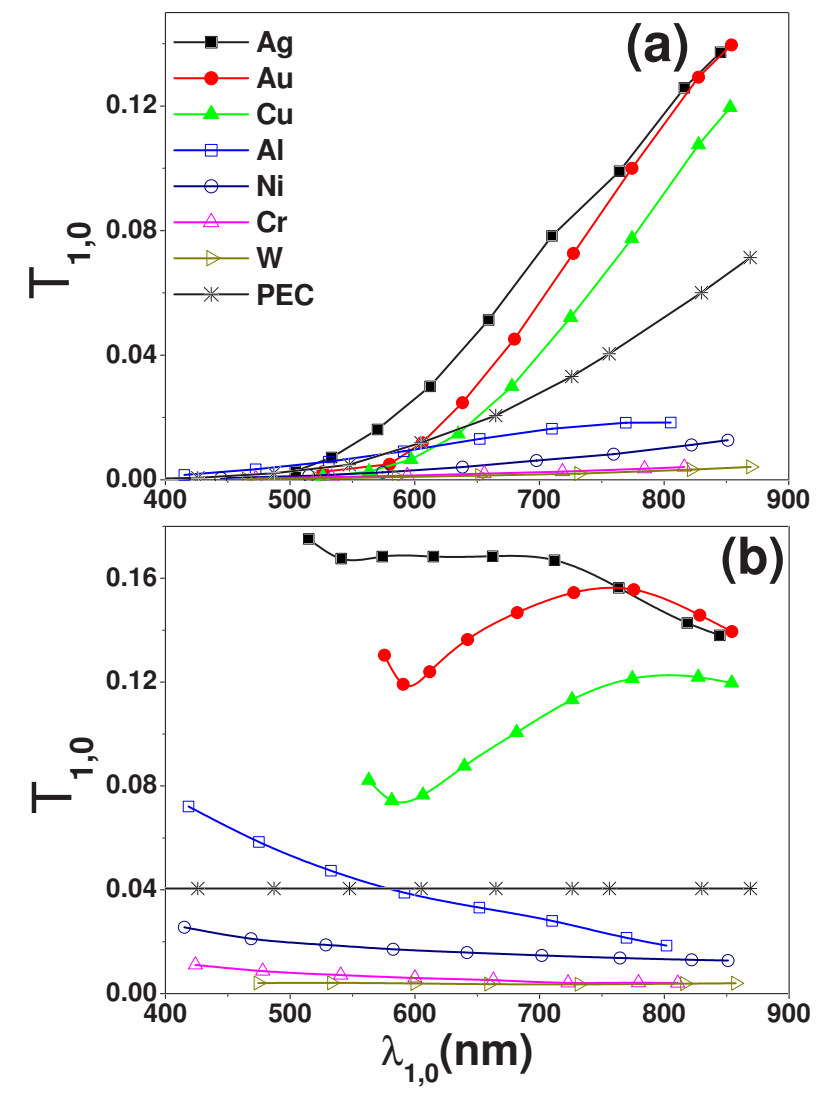

FIG. 7. (Color online) Peak transmittance intensity $T_{1,0}$ as a function of the peak spectral position $\lambda_{1,0}$ for different metals and lattice periods. In both panels, hole diameter is scaled with period as $d=P / 1.75$. In panel (a), metal thickness is kept constant at $w$ $=250 \mathrm{~nm}$. In (b), $w$ is also scaled as $w=P / 2.0$.

Effective areas can then be related to the propagation constants and the EM fields of different modes in the waveguide. ${ }^{35}$ A simpler, phenomenological, approach is simply enlarging the hole radius by a factor (of order unity) times the skin depth. ${ }^{3}$ This is a good approximation, provided the correction (skin depth) is much smaller than the hole radius. In any case, the effective hole area depends on the dielectric constant which, in turn, depends on wavelength. Therefore, from an electromagnetic point of view, even when all nominal lengths are scaled with the period, the "effective" surface percentage covered by holes does not remain constant.

In what follows, we will show how the comparison with the PEC case allows to distinguish which of these two competing mechanisms (absorption and enlargement of the effective area) dominates for a particular circumstance. Let us start with the case of silver. The computed peak transmittance for silver is even larger than the corresponding one in a PEC with the same nominal parameters, as shown in Fig. 7. This suggest that, in this case, the "enlargement of effective area" mechanism is more important than absorption. In order to confirm this point, we have computed the transmittance peak intensities for 2DHA in PEC but with the hole radius enlarged by the skin depth in Ag (computed at the wavelength at which the peak appears). We will refer to this as the "corrected-PEC model." Figure 8 renders the results of these 


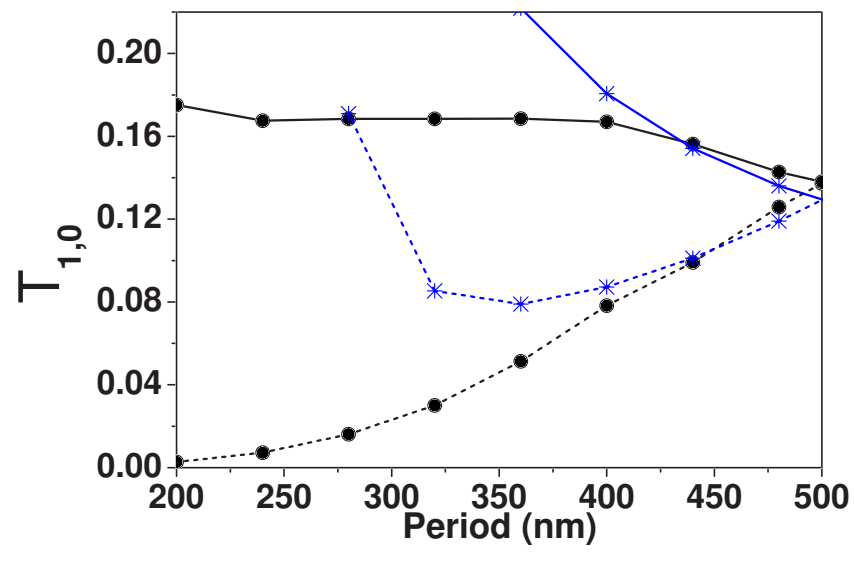

FIG. 8. (Color online) Peak transmittance $T_{1,0}$ as a function of the lattice period for silver (circular symbols) and PEC (asterisks). The solid line represents the case of scaled thickness $(w=P / 2.0)$, while the dashed line is for constant $w=250 \mathrm{~nm}$. In the PEC case, the hole radius was enlarged by one silver skin depth (Fig. 2), evaluated at the corresponding wavelength.

calculations, as well as the corresponding ones for 2DHA in Ag. Discontinuous lines represent the case in which all lengths in the system have been scaled with the period, except for the metal thickness, which has been kept constant at $w=250 \mathrm{~nm}$. The continuous lines render the case where the metal thickness has been also scaled with the period as $w$ $=P / 2$. In all cases, the difference in transmission peak intensities between 2DHA in Ag and the enlarged holes in PEC is less than $30 \%$. Notice that the corrected-PEC model provides much more accurate results at large periods than at smaller ones, which can be associated with the increasing effect of absorption occurring at shorter periods and, correspondingly, smaller wavelengths. This is further corroborated by the fact that, at small periods, the corrected-PEC model overestimates the peak transmittance.

This analysis helps understand the results presented in Fig. 7(b), and therefore the relative importance of the previously described mechanisms for different metals. At peak positions larger than $\lambda_{1,0} \approx 700 \mathrm{~nm}$, the dependence of $T_{1,0}$ on $\lambda_{1,0}$ for $\mathrm{Au}$ is similar to that of $\mathrm{Ag}$, reflecting their similar skin depths and absorption lengths. As $\lambda_{1,0}$ decreases, the difference in skin depths in $\mathrm{Au}$ and $\mathrm{Ag}$ remains constant, but the ratio of their absorption lengths decreases. Correspondingly, due to absorption, $T_{1,0}$ in $\mathrm{Au}$ is smaller than that in $\mathrm{Ag}$ in this frequency regime. At the shorter $\lambda_{1,0}$ computed for $\mathrm{Au}$, this decreasing tendency is reversed, reflecting the increase in the skin depth of $\mathrm{Au}$ (notice that the flattening out of the $\lambda_{1,0}$ vs period curve prevents exploration of shorter values of $\lambda_{1,0}$, see Fig. 4). To summarize, optical transmissions though 2DHA in Ag and Au are similar: In both cases, the resonant transmission is larger than in a PEC with the same nominal parameters, the effect being caused by field penetration in the metal, which effectively enlarges the hole area. Absorption is not the main factor for these metals. On the other hand, 2DHAs in $\mathrm{Cu}$ have transmittance peak characteristics similar to those in $\mathrm{Ag}$ or $\mathrm{Au}$ but with smaller values, reflecting the smaller absorption lengths in $\mathrm{Cu}$ than in the other two noble metals analyzed.

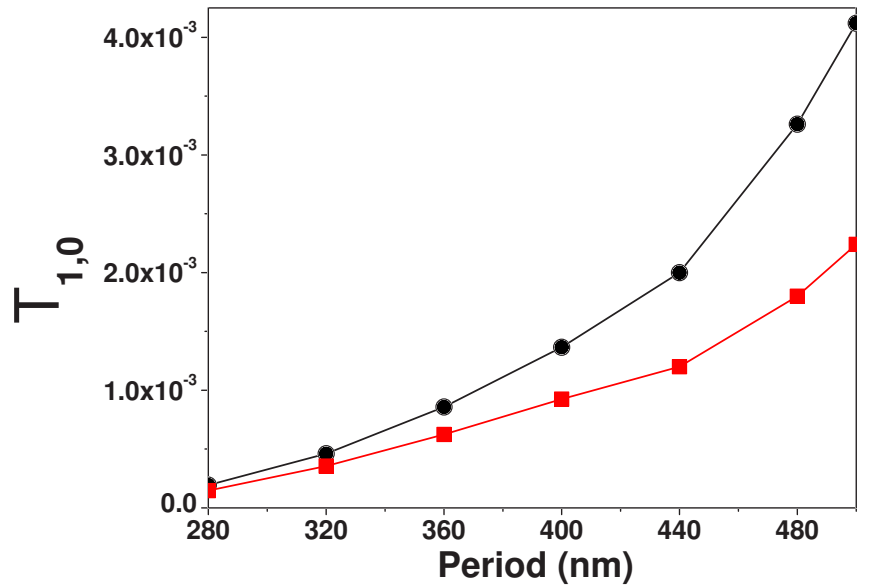

FIG. 9. (Color online) Dependence on period of features of the $S_{1,0}$ peak in W: Maximum (circular symbols) and minimum transmittances (square symbols). All lengths have been scaled as in Fig. 7(a).

A completely different behavior occurs for a 2DHA in $\mathrm{Ni}$ or Cr: In both cases, the peak transmittance is always much smaller than that in Ag (or in a PEC). This behavior occurs although the skin depth in these metals can be even larger than that in $\mathrm{Ag}$ and is due to the large absorption present in both $\mathrm{Ni}$ and $\mathrm{Cr}$.

Hole arrays in $\mathrm{Al}$ have transmittance peak characteristics more similar to the PEC case, reflecting the fact that the skin depth in $\mathrm{Al}$ is, at optical frequencies, much smaller than those of the other metals. Even so, $T_{1,0}$ depends on $\lambda_{1,0}$ for 2DHA in $\mathrm{Al}$ : The skin depth in $\mathrm{Al}$ remains approximately constant with wavelength, implying an "effective area" correction which is relatively smaller at larger hole areas (i.e., larger periods and larger $\lambda_{1,0}$ in the case in which all nominal lengths are scaled). This explains why, in this case, $T_{1,0}$ decreases with $\lambda_{1,0}$ even if the absorption length slightly increases (see Fig. 2). Notice that this behavior of the absorption length in $\mathrm{Al}$ is also very different to that in noble metals, where the absorption length increases strongly with wavelength. As a consequence, the absorption length in $\mathrm{Al}$ is, at $\lambda \approx 750 \mathrm{~nm}$, smaller than that in noble metals. This, combined with the unusually small skin depth, makes $T_{1,0}$ in Al has values of the order of those of bad metals $(\mathrm{Cr}, \mathrm{Ni})$ at the higher end of the spectral window considered.

Finally, a 2DHA perforated in $\mathrm{W}$ presents a very different transmission spectrum. As previously stated, in the considered spectral range, $\mathrm{W}$ is a lossy dielectric. Transmission resonances are aided by Zenneck waves, ${ }^{7,36}$ which are more weakly bound than SPPs. The resonances are much weaker, with a much smaller "visibility": The minima are not very deep and the maxima are not as high as in the case of metals (see Fig. 9). Also, as mentioned before, the spectral position of $T_{1,0}$ in $\mathrm{W}$ (appearing very close to $\lambda_{R}$ ) is different to that of the metallic case.

\section{Full width at half maximum}

The previous analysis on the relative importance of the skin depth and absorption length on the transmittance 

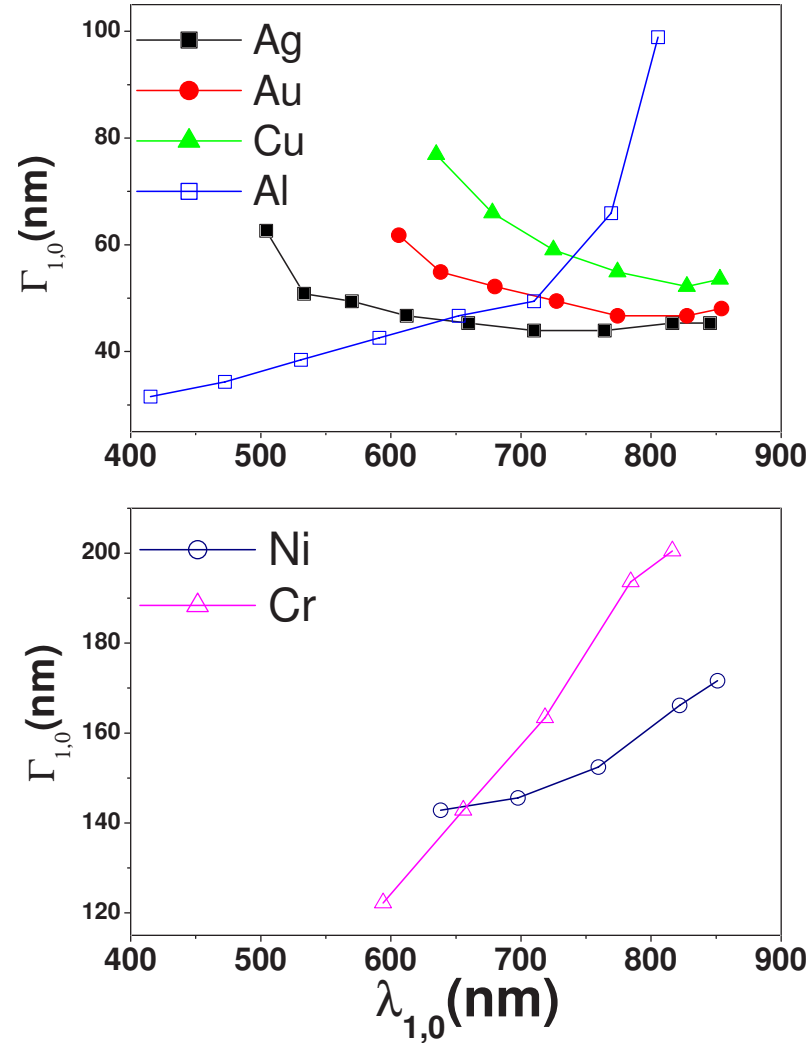

FIG. 10. (Color online) Full width at half maximum for the $S_{1,0}$ peak, $\Gamma_{1,0}$, for 2DHAs in different metals. All lengths have been scaled as in Fig. 7(a), i.e., the metal thickness is kept constant at $w=250 \mathrm{~nm}$.

through 2DHA, based on the peak intensities, is reinforced by the spectral dependence of the peak linewidth. Figure 10 renders $\Gamma_{1,0}$, defined as the full width at half maximum of the $S_{1,0}$ transmittance peak, as a function of $\lambda_{1,0}$. The calculations were performed for the configuration where all lengths are scaled, except the metal thickness which is kept fixed at $w=250 \mathrm{~nm}$. Agreement between experiment ${ }^{21}$ and theory is quite good, although experimental peaks are wider due to finite size effects and/or sample imperfections. The behavior of $\Gamma_{1,0}$ for a $2 \mathrm{DHA}$ in $\mathrm{Au}, \mathrm{Ag}$, and $\mathrm{Cu}$ is similar. In this case and for the parameters considered, radiation is the main loss channel for the surface EM mode, absorption being a (nonnegligible) correction. Absorption is responsible for both the difference in $\Gamma_{1,0}$ between different metals and the decrease of $\Gamma_{1,0}$ with $\lambda_{1,0}$ (following the increase of the absorption length with wavelength).
In the cases of 2DHA in either $\mathrm{Cr}$ or $\mathrm{Ni}, \Gamma_{1,0}$ is much larger than that for noble metals, as expected, given that absorption lengths are much smaller in the former cases. Again, the case of $\mathrm{Al}$ is quite different from that of other metals: It goes from presenting the narrowest resonant peaks at small $\lambda_{1,0}$ to having values of $\Gamma_{1,0}$ of the order of those of bad metals for the larger $\lambda_{1,0}$ considered.

\section{CONCLUSION}

In conclusion, we have investigated theoretically the resonant optical transmission through circular hole arrays drilled in different metals. We have performed two series of calculations. In both of them, all lengths except for the metal thickness are scaled by the same factor. In one of the series, the metal thickness is kept fixed, while in the other, the metal thickness is also scaled. In the first case, for which there are experimental data available, ${ }^{21}$ the comparison between experiment and theory is very good. These results confirm that, in the experiments, the effects of possible inhomogeneities in hole shape and size were small. Also, the metal surface, despite the processing that was received when creating the holes, is described well by a dielectric constant close to its bulk value. Moreover, FDTD is validated as a predictive tool for this kind of systems, as the comparison with experimental data did not require any fitting parameter. The case in which all lengths were scaled allowed the comparison of the transmission properties of real metals with those of a perfect conductor. The analysis reveals different types of behavior of the transmittance in hole arrays in different sets of metals: In $\mathrm{Ag}, \mathrm{Au}$, or $\mathrm{Cu}$, the transmittance is even larger than in the perfect conductor case, reflecting that absorption is low and the penetration of EM fields effectively enlarges the hole area. In $\mathrm{Ni}$ and $\mathrm{Cr}$, although the effective area is as large as in the previous metals, absorption strongly reduces the resonant transmittance. Aluminum behaves very much like a perfect conductor at the lower wavelength end of the optical regime but for peak wavelengths $\lambda_{1,0} \geqslant 700 \mathrm{~nm}$, the resonant transmittance characteristics are dominated by absorption, as in the case of $\mathrm{Ni}$ and $\mathrm{Cr}$. Finally, tungsten, which in the spectral range considered is a dielectric, presents transmission resonances with maxima much smaller than those of even the worse metals $(\mathrm{Ni}, \mathrm{Cr})$.

\section{ACKNOWLEDGMENTS}

The authors acknowledge support from the STREP "Plasmon Enhanced Photonics" (IST-FP6-034506) and the Spanish MECD under Contract No. MAT2005-06608-C02.
${ }^{1}$ T. W. Ebbesen, H. L. Lezec, H. F. Ghaemi, T. Thio, and P. A. Wolff, Nature (London) 391, 667 (1998).

${ }^{2}$ C. Genet and T. W. Ebbesen, Nature (London) 445, 39 (2007).

${ }^{3}$ L. Martín-Moreno, F. J. García-Vidal, H. J. Lezec, K. M. Pellerin,

T. Thio, J. B. Pendry, and T. W. Ebbesen, Phys. Rev. Lett. 86, 1114 (2001).
${ }^{4}$ L. Salomon, F. D. Grillot, A. V. Zayats, and F. de Fornel, Phys. Rev. Lett. 86, 1110 (2001).

${ }^{5}$ P. Lalanne, J. C. Rodier, and J. P. Hugonin, J. Opt. A, Pure Appl. Opt. 7, 422 (2005).

${ }^{6}$ C. Genet, M. P. van Exter, and J. P. Woerdman, Opt. Commun. 225, 331 (2003). 
${ }^{7}$ M. Sarrazin and J.-P. Vigneron, Phys. Rev. E 68, 016603 (2003).

${ }^{8}$ E. Moreno, L. Martín-Moreno, and F. J. García-Vidal, J. Opt. A, Pure Appl. Opt. 8, S94 (2006).

${ }^{9}$ E. Moreno, F. J. García-Vidal, and L. Martín-Moreno, Phys. Rev. B 69, 121402(R) (2004).

${ }^{10}$ B. Wang, W. Dai, A. Fang, L. Zhang, G. Tuttle, T. Koschny, and C. M. Soukoulis, Phys. Rev. B 74, 195104 (2006).

${ }^{11}$ E. Dulkeith, S. J. McNab, and Y. A. Vlasov, Phys. Rev. B 72, 115102 (2005).

${ }^{12}$ J. Christensen, A. I. Fernández-Domínguez, F. de León-Pérez, L. Martín-Moreno, and F. J. García-Vidal, Nat. Phys. 3, 851 (2007).

${ }^{13}$ E. Moreno, A. I. Fernández-Domínguez, J. Ignacio Cirac, F. J. García-Vidal, and L. Martín-Moreno, Phys. Rev. Lett. 95, 170406 (2005).

${ }^{14}$ R. Gordon, A. G. Brolo, A. McKinnon, A. Rajora, B. Leathem, and K. L. Kavanagh, Phys. Rev. Lett. 92, 037401 (2004).

${ }^{15}$ K. J. Klein Koerkamp, S. Enoch, F. B. Segerink, N. F. van Hulst, and L. Kuipers, Phys. Rev. Lett. 92, 183901 (2004).

${ }^{16}$ A. Degiron and T. W. Ebbesen, J. Opt. A, Pure Appl. Opt. 7, S90 (2005).

${ }^{17}$ A. Degiron, H. J. Lezec, N. Yamamoto, and T. W. Ebbesen, Opt. Commun. 239, 61 (2004).

${ }^{18}$ F. J. García-Vidal, E. Moreno, J. A. Porto, and L. Martín-Moreno, Phys. Rev. Lett. 95, 103901 (2005).

${ }^{19}$ A. Mary, S. G. Rodrigo, L. Martín-Moreno, and F. J. GarcíaVidal, Phys. Rev. B 76, 195414 (2007).

${ }^{20}$ H. F. Ghaemi, T. Thio, D. E. Grupp, T. W. Ebbesen, and H. J. Lezec, Phys. Rev. B 58, 6779 (1998).

${ }^{21}$ F. Przybilla, A. Degiron, J.-Y. Laluet, C. Genet, and T. W. Ebbesen, J. Opt. A, Pure Appl. Opt. 8, 458 (2006).

${ }^{22}$ A. Taflove and S. Hagness, Computational Electrodynamics: The
Finite-Difference Time-Domain Method (Artech House, Boston, 2000).

${ }^{23}$ R. J. Luebbers and F. Hunsberger, IEEE Trans. Antennas Propag. 40, 1297 (1992).

${ }^{24}$ L. Martín-Moreno and F. J. García-Vidal, Opt. Express 12, 3619 (2004).

${ }^{25}$ C. T. Chan, Q. L. Yu, and K. M. Ho, Phys. Rev. B 51, 16635 (1995).

${ }^{26}$ A. Vial, A. S. Grimault, D. Macias, D. Barchiesi, and M. L. de la Chapelle, Phys. Rev. B 71, 085416 (2005).

${ }^{27}$ A. D. Rakić, A. B. Djurisisić, J. M. Elazar, and M. L. Majewski, Appl. Opt. 37, 22 (1998).

${ }^{28}$ E. D. Palik, Handbook of Optical Constants of Solids, Academic Press Handbook Series, edited by Edward D. Palik (Academic, New York, 1985).

${ }^{29}$ E. D. Palik, Handbook of Optical Constants of Solids II, edited by Edward D. Palik (Academic, Boston, 1991).

${ }^{30}$ A. Krishnan, T. Thio, T. J. Kim, H. L. Lezec, T. W. Ebbesen, P. A. Wolff, J. B. Pendry, L. Martín-Moreno, and F. J. García-Vidal, Opt. Commun. 200, 1 (2001).

${ }^{31}$ J. B. Pendry, L. Martín-Moreno, and F. J. García-Vidal, Science 305, 847 (2004).

${ }^{32}$ F. J. García-Vidal, L. Martín-Moreno, and J. B. Pendry, J. Opt. A, Pure Appl. Opt. 7, S97 (2005).

${ }^{33}$ F. J. Garcia de Abajo and J. J. Saenz, Phys. Rev. Lett. 95, 233901 (2005).

${ }^{34}$ J. Bravo-Abad, F. J. García-Vidal, and L. Martín-Moreno, Phys. Rev. Lett. 93, 227401 (2004).

${ }^{35}$ F. J. García-Vidal, L. Martín-Moreno, E. Moreno, L. K. S. Kumar, and R. Gordon, Phys. Rev. B 74, 153411 (2006).

${ }^{36}$ M. Sarrazin and J.-P. Vigneron, Phys. Rev. B 71, 075404 (2005). 